\title{
Research Progress on Complexity of Public Bicycle Trip Behavior
}

\author{
Ye Li \\ Beijing Jiao tong University, Beijing 100044, China.
}

351280765@qq.com

\begin{abstract}
With the rapid development of public bicycle systems, it has taken only half a century since the development of the first generation of bicycles in the Netherlands to the fourth generation of bicycle sharing systems supported by information technology. By combing relevant literatures on public bicycle systems both at home and abroad, this paper systematically introduces the research progress and insufficiencies of the public bicycle system's user characteristics, the use of influencing factors, route selection and other public transport modes. Based on literature surveys, we judged: (1) Deepening the sharing of operating model innovations in fixed-site public bicycle systems, (2) Improving public bicycle system-related laws and regulations, (3) Supporting, managing, and monitoring public bicycle systems in an overall strategy. Development, (4) to achieve intelligent, standardized management, will become the main research direction and key areas of public bicycle cycling behavior in the next few years.
\end{abstract}

Keywords: Public bicycles system, user characteristics, use of influencing factors, development hindrance, Future direction.

\section{FOREWORD}

\section{Research Background}

With the development of the global economy, the increase in vehicle ownership has made the greenhouse effect, traffic congestion, environmental pollution, noise pollution and energy shortage more serious. With the acceleration of urbanization, a large number of populations, such as central cities and central cities, radiate, making the road traffic contradiction worse. In many parts of the world, many cities encourage residents to use green and low-carbon travel modes instead of private cars. Local governments are also vigorously promoting the bicycle sharing system (BSS), which is commonly referred to as the public bicycle system. The bicycle sharing system is particularly useful in short-distance travel in big cities and effectively solves the "last mile" problem of travel. In recent years, in some small and medium-sized cities, bicycle sharing systems have gradually begun to be implemented.

\section{Meaning}

Although bicycle sharing systems have a history of more than 50 years, they have received more attention in recent years because they play an important role in reducing the impact of traffic on the environment. The bicycle sharing system provides several important benefits to the city, including improving traffic choices and flexibility, increasing physical activity levels, reducing congestion, transportation costs and air pollution [1], reducing road congestion [2], and reducing carbon emissions. Public bicycles are part of public transportation and can effectively alleviate the "last mile" problem of urban transportation, integrate with public transportation, subway and BRT, and realize "door-to-door" services for public transportation. Second, providing short-distance travel solutions for cities can effectively alleviate the growing pressure on public transportation. 


\section{HISTORY AND DEVELOPMENT STATUS}

\section{Development History}

The concept of "bicycle share" first originated in Europe. The first generation of public bicycle systems began to appear in the Netherlands in 1965. In 1995, Copenhagen, the capital of Denmark, launched the second-generation public self-planning system. At the end of the 1990s, a third-generation public bicycle system appeared in Europe. The public bike rental industry in Europe used computers, unlimited communications, and Internet technologies to achieve digital management and operations. In recent years, many cities have developed a fourth-generation public bicycle system, adopting a modular and mobile service point, and using the solar energy to provide service points, using wireless transmission technology to achieve system information transmission, and making public bicycle systems more Tends to improve.2.2 Development Status

Hangzhou is one of the first cities in China to implement BSS and currently operates the world's largest public bicycle sharing project. [3].

Bicycle sharing is becoming a prominent strategy for solving issues such as the use of clean fuel [4], traffic demand management, land use and transportation links [5].

Since 2010, bicycles have shared more than 88 million bicycles on bike-sharing bikes in the United States. In 2016 alone, riders attracted more than 28 million trips and were higher than the number of visitors to Walt Disney World every year.

\section{USER CHARACTERISTICS}

Although there have been many discussions on feature analysis of bicycle sharing users, vertical research activities seem to lack research on the influence of specific social groups or potential users and actual users. In this context, Ricci $\mathrm{M}$ recommends the use of innovative research methods, taking the historical travel behavior of the user as the research object, supporting bicycle sharing data and socio-economic data, and better understanding the changes in user and non-user travel behavior [6].

\section{Sex}

The phenomenon of gender inequality in the use of bicycles is widespread. Because women have a high degree of aversion to risk [7], there are some deficiencies in bicycle infrastructure and poor road conditions that can reduce the comfort of use. During the use of bicycle [8], women showed stronger route preference [9][10].

After analyzing the data of all trips in New York in August 2017, males accounted for $65.2 \%$ of bicycle users.

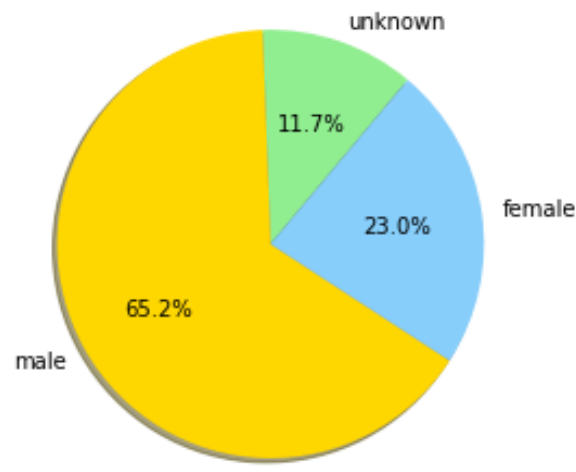

FIGURE 1. User Sex Ratio in the New York. 


\section{Age}

The frequency of use of bicycles also shows a difference with age. In a survey of bicycle users, we found that young people use bicycles more frequently than older people[11].

After analyzing all travel data in New York, USA in August 2017, it was found that people around the age of 30 used bicycles most frequently.

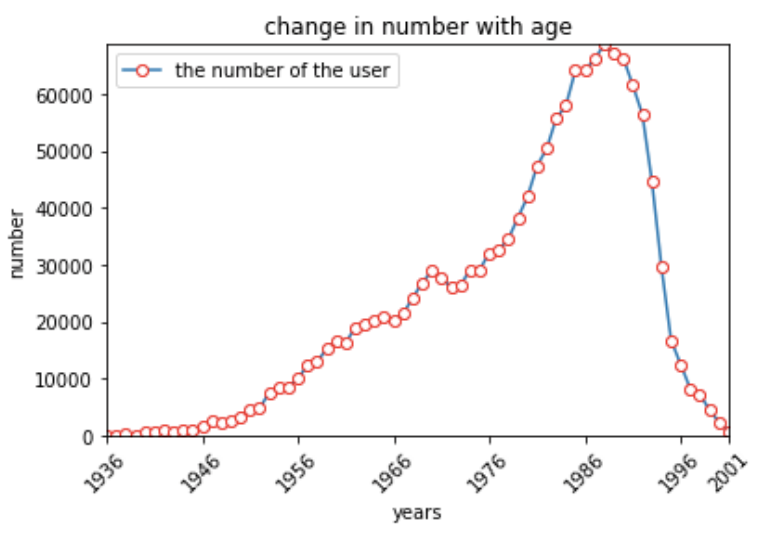

FIGURE 2. Change in Number with Age of the Users

\section{Income}

Users' income will also affect their use behavior to a certain extent. Research in the United States shows that the use of bicycles is negatively correlated with income levels and ownership of vehicles, and the utilization rate of people with lower income is higher [12]. The results of the research in the Netherlands and China are contrary to the fact that the user's income is positively related to the frequency of bicycle use Krygsman [13]and Murphy [14]made similar conclusions on the analysis of bicycle sharing systems in France and Ireland respectively.

\section{Distance of Travel}

Travelers will determine their travel method based on travel distance. In some cities in China where there are less developed public transportation systems and, in some cities, abroad, the travel distance is very short. When walking within $1 \mathrm{~km}$, walks are often used. More people between 2 and $5 \mathrm{~km}$ will prefer to go on bicycles, and more than $5 \mathrm{~km}$ will choose bus transit. And other public travel modes.

In some cities with relatively complete public transportation systems such as Shanghai, most bicycles travel between 0.8 and $1.5 \mathrm{~km} \mathrm{dx}$, less than $0.8 \mathrm{~km}$ walk, and more than $1.5 \mathrm{~km}$, they choose public transport and other means of travel.

\section{Purpose of Travel}

Different travel destinations will have different choices of transportation methods. When traveling to work, the requirements for time are relatively high. They will use a variety of means of transport in a comprehensive manner. At the end of the trip, they will choose public bicycles to travel. When they visit relatives and friends or travel, they will travel. The time requirement is not high, and the requirements for travel comfort are relatively high. Generally, buses, rail transportation, or taxis are used. Bike train combinations are more popular than other uses for travel for work and education purposes [15]. 


\section{INFLUENCING FACTORS}

Bicycle trips have a certain relationship with system properties, station density, weather, geography, and transportation infrastructure.

\section{Toll}

The bicycle sharing system charges are mainly divided into two aspects: rent and deposit.

Caggiani L recommends using the revenue collected by the congestion charging policy to implement a free BSS as a strategy for relieving urban traffic congestion by charging or interfering with links during peak hours [16].

Zhao J used the fare-related variables to explain the use of public bicycles and used the deposit income per capita ratio (DIR) and rent per capita income ratio (PIR) to explain the daily use of PBS in China and the conversion rate [17].

Lin $\mathrm{J}$ J took Taipei PBS and Ubiak as examples. The empirical data shows that whether commuters use PBS is highly dependent on basic charges and basic terms rather than variable costs after the basic period [18].

\section{Climate and Weather}

Another important influencing factor that affects public bicycle rental needs is weather and climate.

Some studies have shown that adverse weather conditions and calendar attributes (working days, weekends, and holidays) have an impact on system use[19], and passenger reduction is related to low temperatures, rainfall, and high humidity.

The results of the study also show that the weather has an impact on the amount of use. Under better weather conditions, long-distance travel is more likely [20].

\section{Other Transportation Methods}

Public bicycles not only need to attract users through their own environmental protection and free features, but also need to take into account the effective connection between ground transportation and rail transit. The purpose of setting up a bicycle sharing system in some cities is to solve the problem of the last kilometer. Its purpose is to connect public transport modes such as public rail transit. Therefore, the convenience of transfer of bicycles and other modes of transportation is an important factor influencing leasing demand. There are mainly the following relationships:

\section{Alternative}

A number of studies have shown that public estimates of $\mathrm{CO} 2$ emission reductions are often exaggerated because only a small percentage of car travel has been replaced by BSS[21]. At the same time, research shows that there is no direct evidence that bicycle sharing significantly reduces traffic congestion, carbon emissions or pollution [22].

\section{Supplementary}

In New York City, the bicycle sharing stations near the subway stations, especially the stations where the average number of subways per month is more, have higher bicycle utilization rates [23]. These findings suggest that there may be a complementary relationship between bicycles and other forms of transportation.

\section{Relevance}

Using a regression analysis, a $10 \%$ increase in bicycle travel was associated with a $2.8 \%$ increase in subway passengers [24]. 


\section{DEVELOPMENT HINDRANCE}

Public bicycles play an important role in alleviating traffic congestion and reducing carbon emissions, but public bicycle systems also face numerous criticisms.

Safety or security is the biggest obstacle preventing more people from riding in the United States [25]. In many cases, accessibility and safety related obstacles restrict the use of bike sharing systems [26, 27].

Even those bicycles sharing systems that are considered to be successful, such as the BIXI service in Montreal, can suffer serious financial difficulties [28].

With the increase in the use of PBS, planners began to notice some deficiencies: there was no customer service hotline, there was no stop sign, and there were not enough bicycles during peak hours [29].

Some of the reasons for the low usage rates in Melbourne and Brisbane include mandatory helmet legislation, a limited network of protected bicycle infrastructure, small watershed scale, poor marketing and promotion, and contracts that do not motivate operators to maximize their use [30] [31].

\section{FUTURE DIRECTIONS}

Based on literature surveys, we judged: (1) Deepening the sharing of operating model innovations in nonstationary public bicycle systems; (2) Improving public bicycle system-related laws and regulations; (3) Supporting, managing, and supervising public bicycle systems in an overall strategy Development and (4) Intelligent and standardized management will become the main research directions and key areas for the study of the complexity of public cycling behavior in the coming years.

\section{REFERENCES}

1. Shaheen S A, Martin E W, Cohen a P, et al. Public bikesharing in North America During a period of rapid expansion: Understanding business models, industry trends \& user impacts: MTI Report 12-29[J]. Mineta Transportation Institute, 2014.

2. Chardon C M D, Caruso G. Estimating bike-share trips using station level data $[\mathrm{J}]$. Transportation Research Part B, 2015, 78:260-279.

3. Elliot Fishman, Simon Washington, Narelle Haworth. Bike Share: A Synthesis of the Literature [J]. Urban Transport of China, 2014, 33(2):148-165.

4. Parkes S D, Marsden G, Shaheen S A, et al. Understanding the diffusion of public bike sharing systems: evidence from Europe and North America [J]. Journal of Transport Geography, 2013, 31(7):94-103.

5. Shaheen S A, Guzman S, Zhang H. Bikesharing in Europe, the Americas, and Asia: Past, Present, and Future [M]// Transportation Research Record: Journal of the Transportation Research Board. 2010:159-167.

6. Ricci M. Bike sharing: A review of evidence on impacts and processes of implementation and operation [J]. Research in Transportation Business \& Management, 2015, 15:28-38.

7. Goodman A, Cheshire J. Inequalities in the London bicycle sharing system revisited: impacts of extending the scheme to poorer areas but then doubling prices [J]. Journal of Transport Geography, 2014, 41(2):272-279.

8. Buehler R, Pucher J. Walking and cycling in Western Europe and the United States: Trends, policies, and lessons [J]. Tr News, 2012:34-42.

9. Aldred R, Elliott B, Woodcock J, et al. cycling provision separated from motor traffic: a systematic review exploring whether stated preferences vary by gender and age [J]. Transport Reviews, 2017, 37(1):29-55.

10. Winters $\mathrm{M}$, Teschke $\mathrm{K}$. Route preferences among adults in the near market for bicycling: findings of the cycling in cities study. [J]. Am J Health Promot, 2010, 25(1):40-47.

11. Smith R G W, Mulamoottil G. The access journey to the railway station and its role in passengers' satisfaction with rail travel [J]. Transport Policy, 2007, 14(5):357-365.

12. Krizek, K., \& Stoebraker, E. (2010). Bicycling and transit: A marriage unre- alized. Transportation Research Record: Journal of the Transportation Research Board, 2144, 161-167.

13. Krygsman, S., \& Dijst, M. (2001). Multimodal trips in the Netherlands: Microlevel individual attributes and residential context. Transportation Research Record: Journal of the Transportation Research Board, 1753, 1119.

14. Murphy, E., Usher, J., 2015. The role of bicycle-sharing in the city: analysis of the Irish experience. Int. J. Sustain. Transport. 9, 116-125. 
15. Givoni M, Rietveld P. The access journey to the railway station and its role in passengers' satisfaction with rail travel [J]. Transport Policy, 2007, 14(5):357-365.

16. Caggiani L, Camporeale R, Ottomanelli M. Planning and Design of Equitable Free-Floating Bike-Sharing Systems Implementing a Road Pricing Strategy [J]. Journal of Advanced Transportation, 2017, 2017:1-18.

17. Zhao J, Deng W, Song Y. Ridership and effectiveness of bikesharing: The effects of urban features and system characteristics on daily use and turnover rate of public bikes in China [J]. Transport Policy, 2014, 35:253-264.

18. Lin J J, Wang N L, Feng C M. Public bike system pricing and usage in Taipei[J]. International Journal of Sustainable Transportation, 2017, 11(4):00-00.

19. Corcoran J, Li T, Rohde D, et al. Spatio-temporal patterns of a Public Bicycle Sharing Program: the effect of weather and calendar events[J]. Journal of Transport Geography, 2014, 41:292-305.

20. Caulfield B, O'Mahony M, Brazil W, et al. examining usage patterns of a bike-sharing scheme in a medium sized city [J]. Transportation Research Part A Policy \& Practice, 2017, 100:152-161.

21. Schwartz S H. Normative influence on altruism [J]. Advances in Experimental Social Psychology, 1977, 10:222-275.

22. Ricci M. Bike sharing: A review of evidence on impacts and processes of implementation and operation [J]. Research in Transportation Business \& Management, 2015, 15:28-38.

23. Noland R B, Smart M J, Guo Z. Bikeshare trip generation in New York City[J]. Transportation Research Part A, 2016, 94:164-181.

24. Erdoğan S, Liu C, Ma T. Bicycle Sharing and Transit: Does Capital Bikeshare Affect Metrorail Ridership in Washington, D.C [J]. Transportation Research Record Journal of the Transportation Research Board, 2015, 2534.

25. Sanders R L. Dissecting Perceived Traffic Risk as a Barrier to Adult Bicycling [J]. Transportation Research Board Annual Meeting, 2013.

26. Sanders R L. Dissecting Perceived Traffic Risk as a Barrier to Adult Bicycling [J]. Transportation Research Board Annual Meeting, 2013.

27. Lathia, N., Ahmed, S., Capra, L., 2012. Measuring the impact of opening the London shared bicycle scheme to casual users. Transp. Res. Part C: Emerg. Technol. 22, 88-102.

28. Béland, D., 2014. Developing sustainable urban transportation: lesson drawing and the framing of Montreal's bikesharing policy. Int. J. Sociol. Soc. Policy 34 (7/8), 545-558.

29. Yao, Y., and Y. Zhou. Bike Sharing Planning System in Hangzhou (in Chinese). Urban Transport of China, Vol. 7, No. 4, 2009, pp. 30-38.

30. Fishman, Fishman, E, Washington, S, Haworth, N \& Watson, A (2015c). Factors influencing bike share membership: an analysis of Melbourne and Brisbane.

31. Transportation Research Part A, 71, 17-30E (2016b). Cycling as transport. Transport Reviews, 36, 1-8. 